\title{
Famotidine and Omeprazole Both Induce Gastric Transmucosal Leak
}

\author{
David Rudolph ${ }^{2}$, Mary Carmen Valenzano ${ }^{1}$, Benjamin $\mathrm{Ngo}^{2}$, Keith Kearney $^{2}$, Giancarlo Mercogliano ${ }^{2}$ \\ and James M. Mullin ${ }^{1,2, *}$
}

${ }^{1}$ Lankenau Institute for Medical Research, Wynnewood, PA 19096, USA

${ }^{2}$ Lankenau Hospital, Department of Medicine, Division of Gastroenterology, Wynnewood, PA, 19096, USA

\begin{abstract}
Background: Our group has recently published that proton pump inhibitors (PPIs) induce transmucosal, paracellular, bidirectional leakage in the gastric corpus epithelium to a wide range of molecules.

Aim: This study was undertaken to determine if $\mathrm{H}-2$ blocker medications also induce such leakage, or whether it is a result of specific inhibition of $\mathrm{H}^{+}, \mathrm{K}^{+}$-ATPase.

Methods: At the beginning and end of a dosage regimen of omeprazole or famotidine, healthy volunteers with no history of gastrointestinal disease consumed a (probe) solution of $100 \mathrm{gms}$ of sucrose in $200 \mathrm{cc}$ of water. Subsequently an $8 \mathrm{hr}$ urine specimen was collected. The sucrose concentration in the urine specimen $(\mathrm{mg} / \mathrm{ml})$ multiplied by the total urine volume equaled the amount of sucrose (mg) which leaked from the gastric lumen into the bloodstream.

Results: Like omeprazole, famotidine was also able to induce significant transmucosal leakage across the mucosal barrier of the upper gastrointestinal tract. Famotidine-induced leakage exhibited a narrower time course than was observed with omeprazole.

Conclusions: The fact that both classes of acid suppressive medications induce leak implies that leak results not from direct inhibition of the $\mathrm{H}^{+}, \mathrm{K}^{+}$-ATPase, or from a side effect of omeprazole-like molecules, but is more generally related to the overall inhibition of acid secretion. The medical significance of such gastric leak is discussed.
\end{abstract}

Keywords: Barrett's esophagus, omeprazole, paracellular, proton pump inhibitor, transepithelial, famotidine.

\section{INTRODUCTION}

The inhibition of acid secretion has been the target for development of a number of medications. The current two major classes, $\mathrm{H} 2$ receptor antagonists and proton pump inhibitors (PPIs), act on the acid secreting gastric parietal cell at very different molecular sites, reflecting their very different molecular structures (Fig. 1). The H2 receptor antagonists (cimetidine, ranitidine, famotidine, and nizatidine) competitively inhibit histamine receptors. Histamine, released by enterochromaffin-like cells, binds to histamine receptors on the surface of parietal cells and stimulates the secretion of acid through its actions on adenylate cyclase. Within the class of $\mathrm{H}-2$ receptor antagonist medications, the onset of acid suppression occurs within one to three hours and the duration of action ranges from 4 to 12 hours [1]. PPIs (omeprazole, lansoprazole, rabeprazole, pantoprazole, esomeprazole), currently one of the largest prescription drug classes in the US, were developed after the realization that the final step of acid secretion was through the enzyme, $\mathrm{H}^{+}, \mathrm{K}^{+}$-ATPase. PPIs are weak bases that become concentrated within the secretory cannuliculi of the parietal cell,

*Address correspondence to this author at the Lankenau Institute for Medical Research, 100 Lancaster Avenue, Wynnewood, PA 19096, USA; Tel: 0+1-484-476-2703 Fax: 0+1+484-476-2205;

E-mail:mullinj@mlhs.org where they are activated to then bind to cysteine residues on the cannulicular luminal surface of the $\mathrm{H}^{+}, \mathrm{K}^{+}$-ATPase [2]. This binding leads to the inhibition of the enzyme. The onset of action begins within an hour of ingestion and the duration of action can be longer than 24 hours. PPIs require prior stimulation of the $\mathrm{H}^{+}, \mathrm{K}^{+}$-ATPase to bind and inactivate it [3].

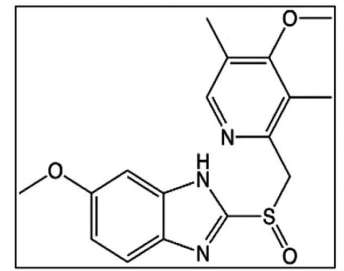

Omeprazole

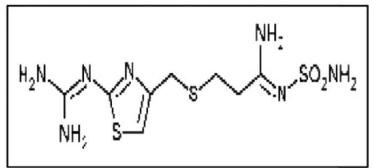

Famotidine

Fig. (1). The widely different structures of famotidine (featuring its 2-guanidinothiazole ring) and omeprazole (with its pyridine ring). 
Recent work from our group has shown in patient-based studies that esomeprazole ( $40 \mathrm{mg} /$ day) causes a transmucosal leak in the lining of the upper gastrointestinal tract in patients presenting for the first time with evidence of reflux disease [4]. The leak was observed to set in quickly after initiation of esomeprazole therapy (3-5 days) and reverse equally quickly after cessation of medication. Subsequent animal model studies, also by our group, showed the following properties of this leak: 1) it is bidirectional; 2) it allows molecules as large as 4,000 daltons to permeate, but not molecules 10,000 daltons or larger; 3 ) it manifests a similar dose dependence to that for inhibition of acid secretion; 4) it occurs near instantaneously if the proton pump inhibitor (PPI) concentration is sufficiently high; 5) it is a general property of PPIs and not specific to only one individual PPI; 6) it requires a luminal fluid $\mathrm{pH}$ below 5.0 [5]. These results confirm and extend the earlier findings of Hopkins et al. (2002) [6] using rat gastric corpus, the first indication that omeprazole was inducing gastric transepithelial leak.

\section{MATERIALS AND METHODOLOGY}

\section{Sucrose Permeability Testing (SPT)}

Sucrose is a disaccharide and therefore lacks a membrane transport-mediated pathway across an epithelial cell. Its only significant route across an epithelial mucosal layer is paracellular, i.e. a frank, macroscopic break in the epithelial lining (e.g. laceration or ulceration) or a molecular-level leak through the gasket-like, tight junctional barrier surrounding each epithelial cell [7]. Once in the bloodstream sucrose cannot be reabsorbed by the kidney for the above reason, and quantitatively arrives in the final urine.

In their homes all patients and healthy controls consumed a chilled solution of $100 \mathrm{~g}$ of sucrose in $200 \mathrm{cc}$ of water containing $5 \mathrm{~g}$ of a citric acid-based flavoring agent at bedtime. After drinking the sucrose solution, an $8 \mathrm{hr}$ (overnight) urine sample was collected in a container with $5 \mathrm{ml}$ of $10 \%$ thymol in isopropanol as antimicrobial preservative. Total urine volume and specific gravity were measured and recorded. The concentration of sucrose in the urine sample was then measured by an enzymatic/spectrophotometric assay after prior desalting of the urine sample by anion and cation exchange resins [8]. Total amount of sucrose in the urine in $\mathrm{mg}$ was determined by multiplying the urine volume in $\mathrm{ml}$ by the sucrose concentration in $\mathrm{mg} / \mathrm{ml}$. This amount of sucrose equates to the number of $\mathrm{mg}$ of sucrose which leaked from the upper GI lumen. Note that since sucrose is enzymatically destroyed by the surface hydrolases in the duodenal lumen (conversion to the monosacharides, glucose and fructose) its leak into the bloodstream must be proximal to this point.

\section{Test Subject Selection}

Test subjects were recruited from clinical and research staff at the performance site. Exclusions from the study were as follows: 1) PPI, H-2 blocker or antacid use in the past 30 days; 2) pregnancy or nursing; 3) liver or kidney disease 4) prior or current gastrointestinal disease other than transient gastroenteritis; 5) prior gastrointestinal surgery other than appendectomy or hemorrhoidectomy; 6) diabetes; 7) unable to provide written, informed consent. Demographic characteristics of test subjects used in these studies are reported in
Table 1. Studies were reviewed and approved by the Lankenau Hospital Institutional Review Board. All test subjects signed an informed consent form kept on file in study offices. Test subjects involved in the omeprazole vs famotidine study were randomly placed in either drug group by the sealed envelope method.

Table 1. Demographics of Test Subject Population Used in the Famotidine/Omeprazole Study

\begin{tabular}{|l|l|l|}
\hline & Famotidine Group & Omeprazole Group \\
\hline \hline Number: & 10 & 10 \\
\hline Age Range: & $36 \pm 4$ (SEM) & $30 \pm 3(\mathrm{SEM})$ \\
\hline Gender Distribution: & $50 \% \mathrm{~F}$ & $10 \% \mathrm{~F}$ \\
\hline Ethnicity Distribution: & $20 \%$ asian $/ 80 \%$ white & $40 \%$ asian $/ 60 \%$ white \\
\hline
\end{tabular}

\section{Statistics}

The sucrose permeability data of the different study populations were compared by paired, Student's t tests. Statistical significance was regarded as a $P$ value less than 0.05 . Data are reported as mean \pm standard error of the mean.

\section{Materials}

The sucrose solutions used to measure permeability were a generous gift of Perk Scientific, Inc. (Yeadon, PA). Enzymatic reagents for determination of urine glucose and sucrose concentrations (invertase, hexokinase, and glucose-6phosphate dehydrogenase) as well as cofactors (ATP and NADP) were products of Sigma Aldrich Chemical Co.

\section{RESULTS}

Given that $\mathrm{H}-2$ receptor antagonists and PPIs both inhibit gastric acid secretion, but do so through very different mechanisms, our group asked whether the induction of gastric leak was peculiar to PPIs or may be a more general side effect of inhibiting acid secretion. Toward this end we compared the effect of $40 \mathrm{mg}$ /day famotidine vs $20 \mathrm{mg}$ /day omeprazole on induction of leak. As previously described, we evaluated gastric leak by the diffusion of (orally consumed) sucrose into the bloodstream and then into the urine [4].

After 5 days of morning doses of famotidine or omeprazole (20 healthy test subjects were randomly assigned [sealed envelope method] to either group), a night time sucrose permeability test on the $5^{\text {th }}$ day showed that omeprazole increased passive leak by $500 \%$ (relative to the pre-drug baseline sucrose leak), a highly significant increase ( $\mathrm{P}<$ 0.01; paired Student's t test, $\mathrm{n}=10$ ), and consistent with our prior results [4]. The famotidine group $(\mathrm{n}=10)$ however showed only a 2 -fold increase which was not statistically significant (Fig. 2). The relatively high variance in the famotidine group specifically, and the $100 \%$ increase in passive leak, led us to speculate however whether famotidineinduced leak may have a narrower time-dependence than the leak produced by PPIs.

We next requested that a second group of healthy controls (a subgroup of the first study group) repeat the above 

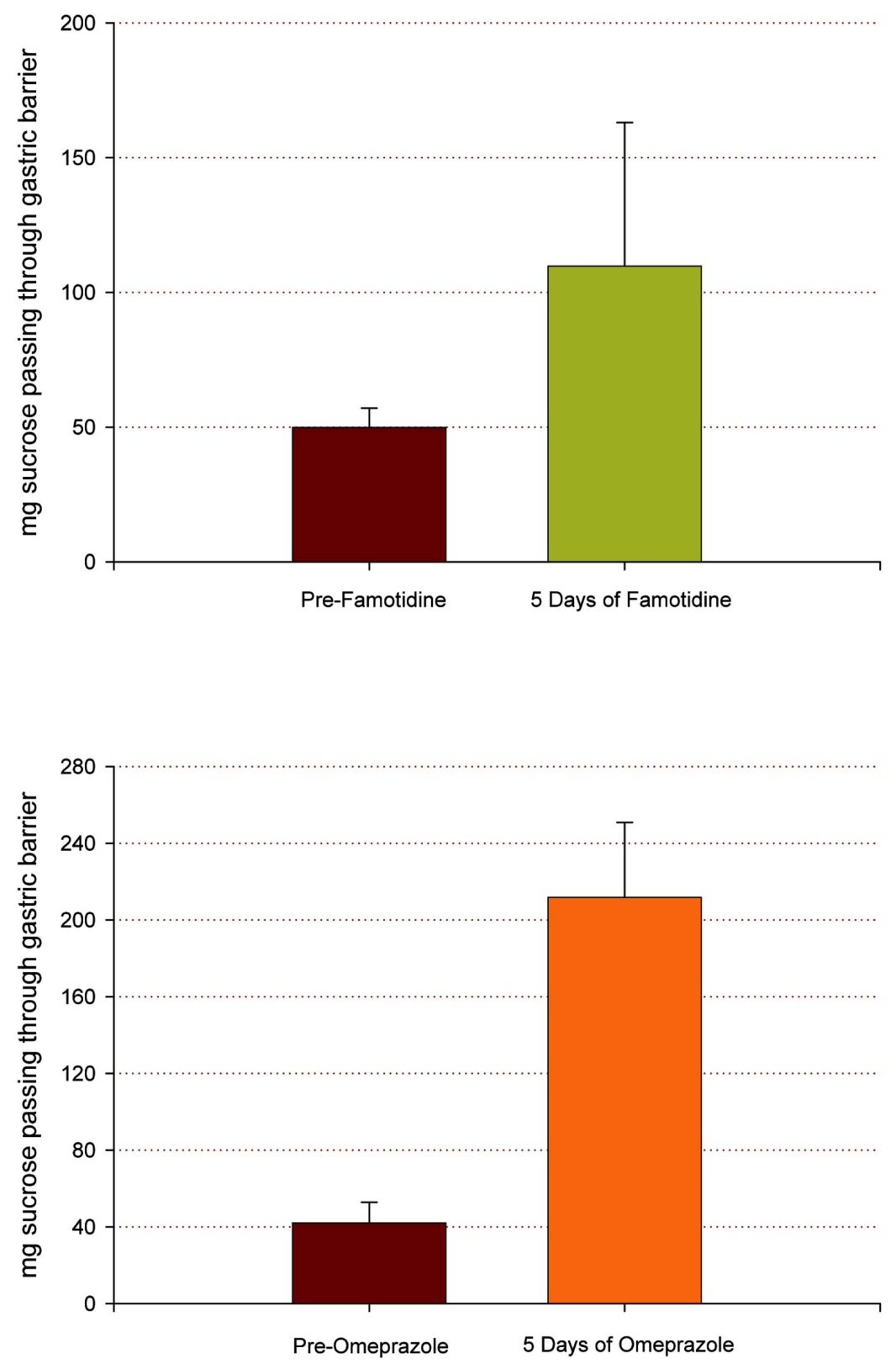

Fig. (2). The increase in gastric leak occurring after 5 days of (morning) doses of omeprazole vs famotidine. Test subjects were given $40 \mathrm{mg}$ of omeprazole or $20 \mathrm{mg}$ of famotidine daily for 5 days as morning doses. Sucrose permeability tests were performed before the medication regimen and immediately after the regimen. Data shown represent the mean \pm SEM for $\mathrm{n}=10$ (different) test subjects. For the omeprazole study, $\mathrm{P}=0.002$ (paired Student's $\mathrm{t}$ test). For the famotidine study, the greater-than- $100 \%$ increase was not quite significant $(\mathrm{P}=0.28)$.

famotidine study with the single difference of taking the famotidine medication between 5 and $6 \mathrm{PM}$ in the evening, rather than a morning dose. This leads to the day 5 famotidine dose being taken only 5-6 hrs prior to the sucrose permeability test rather than the 14-18 hrs that would occur with a morning famotidine dosing regimen. As shown in Fig. (3), this change in dosing schedule increased observed leak to near 3-fold greater than baseline level, a change that was now statistically significant $(\mathrm{P}=0.03$, paired Student's t test, $\mathrm{n}=5)$. This apparent narrower window for famotidineinduced gastric leak (as opposed to PPI-induced gastric leak) parallels the above-mentioned temporally narrower window for $\mathrm{H}-2$ receptor antagonist acid suppression compared to PPI-acid suppression.

In a final study, we addressed the time frame of induction of gastric leak, in terms of the appearance of leak from the time a patient begins to take medication. Again a subgroup of the group used in the first study, was used here. We previously reported that PPI-induced leak begins as early as 3-5 days after beginning PPI medication [4]. Table 2 reveals that a similar time frame exists for famotidine. In this study, leak increased significantly above baseline levels as early as day 3 , paralleling the result reported earlier with PPIs. 


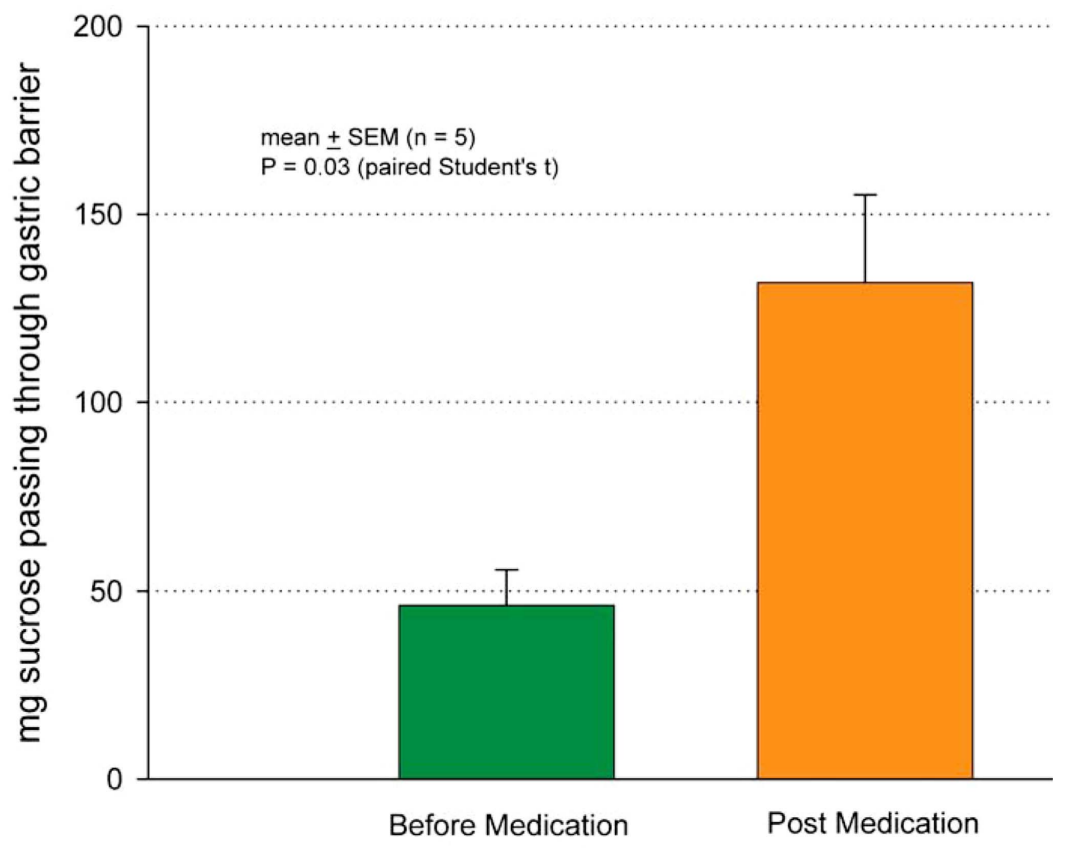

Fig. (3). The increase in gastric leak occurring after 5 days of morning vs evening doses of 20 mg famotidine. Data represent the mean \pm SEM for $n=5$ test subjects. $P=0.03$ (paired Student's $t$ test) for the evening dosing schedule vs. P $>0.05$ (Not Significant) for the morning dosing schedule (Fig. 2).

Table 2. The Increase in Gastric Leak after 3, 5 and 7 Days of Famotidine Exposure

\begin{tabular}{|l|l|l|}
\hline Condition & Sucrose Leak (mg) & Number of Test Subjects \\
\hline \hline Pre-Famotidine & $42.4+7.5$ & 15 \\
\hline 3 Days of Famotidine & $106.3+30.7 *$ & 05 \\
\hline 5 Days of Famotidine & $211.8+39.0 *$ & 10 \\
\hline 7 Days of Famotidine & $88.8+33.1 * *$ & 05 \\
\hline
\end{tabular}

Data represents the mean \pm SEM of test subjects who performed up to a 7 day course of $20 \mathrm{mg}$ famotidine with periodic sucrose permeability tests. The number in parentheses indicates the number of test subjects performing the specific study time point. (* indicates $\mathrm{P}<0.01$; ** indicates $\mathrm{P}<0.05$ [Student's $\mathrm{t}$ test] relative to the prefamotidine sucrose leak level. The 3,5 and 7 day levels of leak were not significantly different from each other $(\mathrm{P}>0.05)$.

\section{DISCUSSION AND CONCLUSION}

Our group is currently exploring the properties of this induced gastric leak in terms of the types of molecules that can and cannot permeate through it. As described above, one of its already-reported properties is that only molecules smaller than 4000 daltons can pass [5]. This statement however does not take into account the complexities of the molecule's charge, shape and hydrophobicity, all of which can prevent its permeation even though it may be sufficiently small in overall mass. It is specifically the types of molecules able to permeate that will determine the medical relevance of the leak. If, e.g. luminal antigens are able to permeate, that creates one type of biomedical repercussion by generating conditions for an inflammatory cascade in that tissue. If, e.g. certain oral medications with narrow therapeutic windows are able to pass across the GI barrier, that generates important implications for the pharmacokinetics of medications taken orally while on PPI-therapy. A recent clinical report of PPI-digoxin drug interaction suggests that the concern here may need to extend beyond merely the well known liver cytochrome inhibition by certain PPIs [9]. Prior work from our group has in fact shown that digoxin can permeate through this gastric leak pathway (although the similar size drug, phenytoin, cannot permeate) [10]. Lastly, if newgeneration 'biological' drugs (peptides, oligonucleotides) are able to diffuse through the PPI-induced leak, this creates a drug delivery 'opportunity' that might permit oral forms of medications that must normally be injected or i.v. infused. We recently reported that the 8 -mer peptide, bradykinin, is able to diffuse intact across the gastric corpus mucosa in the presence of PPI drugs [11].

The finding that omeprazole and famotidine both induce gastric leakage yet have quite different molecular targets, suggests that it is the inhibition of acid secretion (that which PPIs and H-2 receptor blockers share in common) that is mediating the induction of a paracellular leak. In this regard the recent report that extracellular $\mathrm{pH}$ buffering can induce changes in epithelial tight junctions is noteworthy [12]. It is thus possible that dramatically altering the $\mathrm{pH}$ of the unstirred layer at the apical surface of the gastric epithelia by inhibiting their secretion of acid, may induce a change in gastric epithelial tight junctions that increases paracellular leak.

Since H-2 blockers act on the extracellular surface of the histamine receptor (basal-lateral cell membrane) whereas PPIs act on an intracellular site of the H,K-ATPase (canalicular and apical cell membrane), they share in common only the final effect - inhibition of acid secretion with consequent elevation of luminal $\mathrm{pH}$. Realization that it is the luminal $\mathrm{pH}$ elevation-effect of PPIs and H-2 blockers that somehow is involved in paracellular leak induction is a remarkably useful clue to mechanism of action. It signifies that PPIs are not inducing the leak by a side effect on molecular targets separate from the acid-secretion pathway, since PPIs 
and H-2 blockers would be unlikely to share such an unrelated molecular target in common. This manuscript's central finding does therefore argue for inhibition of acid secretion and perhaps elevation of luminal $\mathrm{pH}$ as the trigger for this leak induction.

One well known concern about chronic PPI use has been that the elevation of luminal $\mathrm{pH}$ may allow for increased survival of luminal pathogens, thereby creating more infectious disease opportunities. A similar concern, and one that may perhaps derive from the PPI-induced paracellular leak, is that PPI use may increase the incidence of food allergies [13]. The combination of increased luminal $\mathrm{pH}$ (with less acid digestion of antigenic food proteins) and induction of an unregulated transmucosal leak pathway may provide the mechanism.

\section{ACKNOWLEDGMENTS}

\section{Funding Source}

Sharpe-Strumia Research Foundation of the Bryn Mawr Hospital.

\section{CONFLICT OF INTEREST DISCLOSURE}

None.

\section{REFERENCES}

[1] Feldman M, Burton ME. Histamine2-receptor antagonists. Standard therapy for acid-peptic diseases. 1. N Engl J Med 1990; 323: $1672-80$
[2] Sachs G, Wallmark B. Biological basis of omeprazole therapy. J Gastroenterol Hepatol 1989; 4 Suppl 2: 7-18.

[3] Wolfe MM, Sachs G. Acid suppression: optimizing therapy for gastroduodenal ulcer healing, gastroesophageal reflux disease, and stress-related erosive syndrome. Gastroenterology 2000; 118: S9-31.

[4] Mullin JM, Valenzano MC, Whitby M, et al. Esomeprazole induces upper gastrointestinal tract transmucosal permeability increase. Aliment Pharmacol Ther 2008; 28: 1317-25.

[5] Murray LJ, Gabello M, Rudolph DS, et al. Transmucosal gastric leak induced by proton pump inhibitors. Dig Dis Sci 2009; 54: 1408-17.

[6] Hopkins AM, McDonnell C, Breslin NP, O'Morain CA, Baird AW. Omeprazole increases permeability across isolated rat gastric mucosa pre-treated with an acid secretagogue. J Pharm Pharmacol 2002; 54: 341-7.

[7] Meddings JB, Wallace JL, Sutherland LR. Sucrose Permeability: A Novel Means of Detecting Gastroduodenal Damage Noninvasively. Am J Ther 1995 2: 843-9.

[8] Bergmeyer H, Bernt E. Sucrose, in Methods of Enzymatic Analysis. 2nd Edition ed. New York: Academic Press 1974.

[9] Kiley CA, Cragin DJ, Roth BJ. Omeprazole-associated digoxin toxicity. South Med J 2007; 100: 400-2.

[10] Gabello M, Valenzano MC, Zurbach EP, Mullin JM. Omeprazole induces gastric transmucosal permeability to the peptide bradykinin. World J Gastroenterol 2010; 16: 1097-103.

[11] Gabello M, Valenzano MC, Barr M, Zurbach P, Mullin JM. Omeprazole Induces Gastric Permeability to Digoxin. Dig Dis Sci 2010; 55: 1255-63.

[12] Helms HC, Waagepetersen HS, Nielsen CU, Brodin B. Paracellular Tightness and Claudin-5 Expression is Increased in the BCEC/ Astrocyte Blood-Brain Barrier Model by Increasing Media Buffer Capacity During Growth. AAPS J 2010; 12: 759-70..

[13] Untersmayr E, Scholl I, Swoboda I, et al. Antacid medication inhibits digestion of dietary proteins and causes food allergy: a fish allergy model in BALB/c mice. J Allergy Clin Immunol 2003; 112: $616-23$

This is an open access article licensed under the terms of the Creative Commons Attribution Non-Commercial License (http://creativecommons.org/licenses/ by-nc/3.0/) which permits unrestricted, non-commercial use, distribution and reproduction in any medium, provided the work is properly cited. 